\title{
QUARK HADRON DUALITY TESTS ON POLARIZED STRUCTURE FUNCTIONS USING CLAS
}

\author{
T.A. FOREST * \\ Center for Applied Physics Studies \\ P.O. Box 10348 \\ Ruston, LA 71270, USA \\ E-mail: tforest@jlab.org \\ FOR THE CLAS COLLABORATION
}

\begin{abstract}
Inclusive electron-nucleon scattering data from Jefferson Lab's Hall B has been analyzed to test quark-hadron duality for the polarized structure function $g_{1}\left(x, Q^{2}\right)$ over a $Q^{2}$ range from 0.2 to $3.5 \mathrm{GeV}^{2} / \mathrm{c}^{2}$. Incident polarized electron beam energies of 1.6 and $5.7 \mathrm{GeV}$ were scattered by polarized ${ }^{15} \mathrm{NH}_{3}$ and ${ }^{15} \mathrm{ND}_{3}$ targets. The measurements of $g_{1}\left(x, Q^{2}\right)$ in the resonance region appear to be equivalent to a fit of $g_{1}\left(x, Q^{2}\right)$ in the deep inelastic scattering region at high $Q^{2}$. A quantitative test comparing the ratio of first moment in the resonance region to the first moment in the deep inelastic region is consistent with unity when $Q^{2}>2.0 \mathrm{GeV}^{2} / c^{2}$ but substantially departs from unity when $Q^{2}<1.0 \mathrm{GeV}^{2} / \mathrm{c}^{2}$.
\end{abstract}

\section{Introduction}

The theoretical description of baryon-baryon and baryon-lepton interactions has typically utilized quark-gluon degrees of freedom for high energy interactions and hadronic degrees of freedom at low energies. In 1970, Bloom and Gilman experimentally observed ${ }^{1}$ that electroproduction measurements of the nucleon resonance region structure function $\left(\nu W_{2}\left(x, Q^{2}\right)\right)$, at low values of the four momentum transfer squared $\left(0.4<Q^{2}<2.4\right.$ $\left.\mathrm{GeV}^{2} / \mathrm{c}^{2}\right)$, were equivalent to measurements of the structure function $F_{2}(x)$ made at $Q^{2} \approx 7 \mathrm{GeV}^{2} / \mathrm{c}^{2}$, in the deep inelastic scattering (DIS) region, when the resonance data were averaged over the same range in scaling variable $\omega^{\prime}=1+W^{2} / Q^{2}$ where $\mathrm{W}$ represents the invariant mass. To explain the experimental observations made by Bloom and Gilman, A. de Rújula, H. Georgi and H.D. Politzer employed a perturbative operator product ex-

$\overline{{ }^{*} \text { Work partially supported by grant }}$ award \#0244998 of the National Science Foundation. 
pansion of QCD structure function moments ${ }^{2}$. Higher twist corrections (initial and final state interactions between the struck quark and target remnant) appeared to be sufficiently small or were canceling such that the values of the lower moments of $F_{2}$, averaged over a sufficient range in $x$, were the same over a range of $Q^{2}$. This dual nature is commonly referred to as duality.

The data in Reference ${ }^{3}$ extended the Bloom and Gilman measurements and utilized a quantitative comparison by testing the veracity of the expression

$$
\int_{\xi_{\min }}^{\xi_{\max }} \nu W_{2}(\xi) d \xi=\int_{\xi_{\min }}^{\xi_{\max }} F_{2}(\xi) d \xi
$$

where the Nachtmann ${ }^{4}$ variables $\xi_{\text {min }}$ and $\xi_{\text {max }}$ are determined by the invariant mass cuts of $2 \mathrm{GeV}$, the conventional resonance-DIS border, and $1.15 \mathrm{GeV}$, pion threshold. Equation 1 was tested as a function of $Q^{2}$ by using the data to evaluate the left hand side and three different models ${ }^{5},{ }^{6},{ }^{7}$ to evaluate the right hand side. Equation 1 was shown to be valid at the $10 \%$ level for $Q^{2}>1 \mathrm{GeV}^{2} / \mathrm{c}^{2}$. If contributions from elastic scattering are included, then Equation 1 was found to hold at the same level down to $Q^{2}=0.2 \mathrm{GeV}^{2} / \mathrm{c}^{2}$.

Duality for the polarized structure function $g_{1}\left(x, Q^{2}\right)$ has also been investigated using

$$
\int_{x_{\min }}^{x_{\max }} g_{1}^{r e s}\left(x, Q^{2}\right) d x=\int_{x_{\min }}^{x_{\max }} g_{1}^{D I S}\left(x, Q^{2}\right) d x .
$$

The veracity of Equation 2 may be evaluated by constructing a ratio of the resonance integral to the DIS integral. The HERMES collaboration ${ }^{8}$ evaluated this ratio for $g_{1}\left(x, Q^{2}\right)$ using the relation

$$
g_{1}\left(x, Q^{2}\right)=A_{1}(x) F_{2}\left(x, Q^{2}\right)[2 x / \xi-1] /\left[2 x 1+R\left(x, Q^{2}\right)\right]
$$

where measurements of $A_{1}\left(x, Q^{2}\right)$ in the resonance region were used on the left hand side of Equation 2 and a $Q^{2}$ independent fit to the DIS data for $A_{1}$ was used for the right hand side of Equation 2. $F_{2}\left(x, Q^{2}\right)$ was taken from parameterizations of resonance ${ }^{9}$ and DIS data ${ }^{10}$. The value of $R\left(x, Q^{2}\right)$ was chosen to be 0.18 over the whole resonance region while in the DIS region, defined as $W>2.0 \mathrm{GeV}, R\left(x, Q^{2}\right)$ was taken from Reference ${ }^{11}$. The data were grouped into three $Q^{2}$ bins of about $1.5,3$, and $5 \mathrm{GeV}^{2} / \mathrm{c}^{2}$ between $1.2 \mathrm{GeV}^{2} / \mathrm{c}^{2}$ and $12 \mathrm{GeV}^{2} / \mathrm{c}^{2}$. Equation 2 was found to hold to within $20 \%$ at the lowest $Q^{2}$ bin and no large effects due to target mass corrections were reported. 
The Cebaf Large Acceptance Spectrometer (CLAS) collaboration's EG1 run group at Jefferson Lab has completed taking data to measure polarized structure functions in Hall B. The tests of quark-hadron duality reported here are based on an analysis of the data taken at incident longitudinally polarized electron energies of $1.6 \mathrm{GeV}$ and $5.7 \mathrm{GeV}$. The CLAS facilitates a kinematic coverage from 0.2 to $3.5 \mathrm{GeV}^{2} / \mathrm{c}^{2}$ in $Q^{2}$ and $x$ from 0.1 to 0.5 for this data set.

\section{Apparatus}

A detailed description of the CLAS may be found in Reference ${ }^{12}$. The spectrometer is equipped with a superconducting magnet and three drift chamber regions ${ }^{13}$ which cover roughly $80 \%$ of the azimuthal angles and reconstruct the momentum of a charged particle which scatters within a polar angular range between $8^{\circ}$ and $142^{\circ}$. An array of scintillator counters ${ }^{14}$ covers the above angular range and is used to determine the time of flight for charged particles $\mathrm{A}$ forward angle electron calorimeter ${ }^{15}$, 16 radiation lengths thick, exists up to a polar angle of $45^{\circ}$ and is used along with the drift chambers to separate pions from electrons for this analysis. A Cerenkov detector ${ }^{16}$ covers the same angular range as the calorimeter and is used in conjunction with the calorimeter to create a coincidence trigger.

The polarized structure function data were collected using ammonia $\left({ }^{15} \mathrm{NH}_{3}\right)$ and deuterated ammonia $\left({ }^{15} \mathrm{ND}_{3}\right)$ targets, polarized via Dynamic Nuclear Polarization (DNP $)^{17}$, in conjunction with longitudinally polarized electrons ranging in energy from $1.6 \mathrm{GeV}$ up to $5.7 \mathrm{GeV}$. Although the incident electron polarization and target polarization were monitored by a Moller polarimeter and Nuclear Magnetic Resonance (NMR) system respectively during the experiment, the measured elastic scattering asymmetry observed from events contained within the same data set as inelastic scattering events, a feature of the large acceptance detector, were used to determine the product of beam and target polarization $\left(P_{b} \times P_{t}\right)$. The measured product of $P_{b} \times P_{t}$ for the $1.6 \mathrm{GeV}$ data set averaged to $60.0 \pm 2.7 \%$ using the ${ }^{15} \mathrm{NH}_{3}$ target and $18.5 \pm 0.3 \%$ using the ${ }^{15} \mathrm{ND}_{3}$ target. The data taken using a $5.7 \mathrm{GeV}$ incident electron had an average value for $P_{b} \times P_{t}$ of $55.6 \pm 3.9 \%$ using the ${ }^{15} \mathrm{NH}_{3}$ target and $23.2 \pm 3.3 \%$ using the ${ }^{15} \mathrm{ND}_{3}$ target. 


\section{Preliminary Results}

The polarized structure function $g_{1}\left(x, Q^{2}\right)$ as a function of the Nachtmann scaling variable $\xi$ is shown for the proton in Figure 1 and for the deuteron in Figure 2. Unlike the method used in Reference ${ }^{8}, g_{1}\left(x, Q^{2}\right)$ is extracted from the measured double spin asymmetry $A_{\|}$such that

$$
g_{1}\left(x, Q^{2}\right)=\frac{\tau}{1+\tau}\left[\frac{A_{\|}}{D}+\left(\frac{1}{\sqrt{\tau}}-\eta\right) A_{2}\left(x, Q^{2}\right)\right] F_{1}\left(x, Q^{2}\right)
$$

where $\tau \equiv \nu / Q^{2}, D=\frac{1-(1-y) \epsilon}{1+\epsilon R\left(x, Q^{2}\right)}, F_{1}\left(x, Q^{2}\right)$ represents an unpolarized structure function, and $R\left(x, Q^{2}\right)$ is the ratio of the longitudinal photoabsorbption crosssection $\left(\sigma_{L}\right)$ to the transverse $\left(\sigma_{T}\right)^{18} . y \equiv \nu / E$ is the fractional energy loss of the incident electrons, $\nu$ is the energy transfered to the target $\left(E-E^{\prime}\right)$ and $\epsilon$ is the magnitude of the virtual photon's longitudinal polarization given by

$$
\epsilon=\left[1+2\left(1+\nu^{2} / Q^{2}\right) \tan ^{2}(\theta / 2)\right]^{-1}
$$

where $\theta$ is the electron scattering angle. The double spin asymmetry $A_{\|}$is defined as

$$
A_{\|}=\frac{\sigma_{\uparrow \downarrow}-\sigma_{\uparrow \Uparrow}}{\sigma_{\uparrow \downarrow}+\sigma_{\uparrow \Uparrow}}
$$

In this analysis, the functions $R\left(x, Q^{2}\right), F_{1}\left(x, Q^{2}\right)$ and $A_{2}\left(x, Q^{2}\right)$ were extracted from fits to the present world data set ${ }^{19}$. The parametrization of $R\left(x, Q^{2}\right)$ is an update to the model used in Reference ${ }^{8}$ and included data from recent measurements in the proton resonance region ${ }^{20}$.

The structure function $g_{1}\left(x, Q^{2}\right)$ is predominantly negative for both the proton and deuteron at values of $Q^{2}<1 \mathrm{GeV}^{2} / \mathrm{c}^{2}$ and values of $\xi$ which kinematically correspond to the $\Delta(1232)$ resonance. This observation is consistent with our description of the $\Delta(1232)$ resonance as a spin $3 / 2$ state. In the limit of infinite $Q^{2}$, the structure function $g_{1}\left(x, Q^{2}\right)$ is proportional to the asymmetry $A_{1}$ and $F_{1}$ such that $A_{1} \propto \sigma^{1 / 2}-\sigma^{3 / 2}$ ( if $g_{2}$ and $A_{2}$ are sufficiently small) and $F_{1}$ is an unpolarized structure function. The coupling of a spin 1 virtual photon and a spin $1 / 2$ nucleon to a spin $3 / 2 \Delta(1232)$ results in a smaller amplitude for a total spin $1 / 2$ z-projection $\left(S_{z}=1 / 2\right)$ final state than $S_{z}=3 / 2$ and as a result the difference $\sigma^{1 / 2}-\sigma^{3 / 2}$ is expected to be negative. As $Q^{2}$ becomes substantially larger than unity though, the data indicate that $g_{1}\left(x, Q^{2}\right)$ moves closer to zero and may even be positive in a kinematic region which corresponds to 
the $\Delta(1232)$ resonance. As a result of this behavior, the generalized GDH integral $^{21}$ for the proton becomes negative as $Q^{2}$ falls below $0.5 \mathrm{GeV}^{2} / \mathrm{c}^{2} 22$. Alternatively, the contribution of the $\Delta(1232)$ resonance to the generalized GDH integral wanes as $Q^{2}$ increases. One may expect the $\Delta(1232)$ to play a similar role in Equation 2 to the point that the equality is not maintained unless an equally apposing contribution, such as the elastic contribution, is included.

To test duality, Equation 2 is cast in the form

$$
I^{r e s} / I^{D I S} \equiv \frac{\int_{x_{\min }}^{x_{\max }} g_{1}^{r e s}\left(x, Q^{2}\right) d x}{\int_{x_{\min }}^{x_{\max }} g_{1}^{D I S}\left(x, Q^{2}\right) d x}
$$

The integrations limits $x_{\min }$ and $x_{\max }$ are determined by the measured invariant mass $(W)$ limits for a given $Q^{2}$ bin. The ratio $\left(I^{\text {res }} / I^{D I S}\right)$ is shown in Figure 3. The EG1 data are in general agreement with the HERMES data, but provide a much more precise and detailed picture of the onset of duality and may be used to investigate local duality as well. The EG1 data show that the ratio test begins to fail when $Q^{2}$ becomes less than 1 $\mathrm{GeV}^{2} / \mathrm{c}^{2}$. Equation 2 is valid within $10 \%$ for the proton and $30 \%$ for the deuteron after $Q^{2}$ goes beyond $2 \mathrm{GeV}^{2} / \mathrm{c}^{2}$.

\section{Summary}

Measurements of the polarized structure function $g_{1}\left(x, Q^{2}\right)$ have illustrated the principle of quark-hadron duality for values of $Q^{2}$ above $2.0 \mathrm{GeV}^{2} / \mathrm{c}^{2}$ for the proton and deuteron but substantially depart from this principle when $Q^{2}$ is less than $1 \mathrm{GeV}^{2} / \mathrm{c}^{2}$. The common conjecture is that higher order terms in the perturbative QCD twist expansion, which are expected to dominate as $x \rightarrow 1$, are instead canceling or very small in kinematic regions where duality holds ${ }^{25}$. Reference ${ }^{25}$ further asserts that contributions from dynamical Higher Twists become large and negative at low $x$ and $Q^{2}$ unlike the unpolarized structure functions. This may be one reason why the onset of duality for $g_{1}\left(x, Q^{2}\right)$ occurs at substantially larger values of $Q^{2}$ than the unpolarized structure function $F_{2}$. Additional data at beam energies of 2.4 $\mathrm{GeV}$ and $4.2 \mathrm{GeV}$ are underway and should improve the precision of these measurements at moderate $Q^{2}$.

\section{References}

1. E.D. Bloom and F.J. Gilman,Phys. Rev. Let., 25 \#16, 1140-1143 (1970).

2. A. de Rújula, H. Georgi and H.D. Politzer,Ann. Phys. 103, 315 (1975); H. Georgi and H.D. Politzer, Phys. Rev.. D 14, 1829 (1976); 
3. I. Niculescu, et. al. Phys. Rev. Let., 85 \#6, 1186-1189(1999).

4. O. Nachtmann, Nucl. Phys.,63, 237 (1975)

5. A. Milsztajn et al., Z. Phys. C 49 (1991) 527.

6. A.D. Martin, et.al.,Phys. Rev., B387,419,1996, hep-ph/9409257

7. H.L. Lai, et.al., Phys. Rev. D51 (1995) 4763-4782

8. A. Airapetian et. al.,Phys.Rev.Lett. 90, 092002 (2003).

9. A. Bodek, et. al., Phys. Rev. D 20, 1471, (1979).

10. K. Abe, et. al., Phys. Rev. D 58, 112003, (1998)

11. L. W. Whitlow, et. al., Phys. Lett., B 250, 193 (1990).

12. B.A. Meking, et. al., Nucl. Instr. Meth., 503, 513 (2003)

13. M.D. Mestayer, et. al., Nucl. Instr. Meth., A449, 81 (2000)

14. E.S. Smith, et. al., Nucl. Instr. Meth., A432, 265 (1999)

15. M. Amarian, et. al., Nucl. Instr. Meth., A460, 239 (2001)

16. G. Adams, et. al., Nucl. Instr. Meth., A465, 414 (2001)

17. C.D. Keith, et. al., Nucl. Instr. Meth. Phys. Res., Sect. A501, 327 (2003)

18. "The Structure of the Proton", R.G. Roberts, Cambridge University Press, 1990 (ISBN 0-521-35159-6)

19. S.E. Kuhn, private communication.

20. C.E. Keppel, proceedings SPIN2002 conference, 2002, New York.

21. X. Ji and J. Osborne, J. Phys. G:Nucl. Part. Phys., 27,127 (2001)

22. G.E. Dodge, these proceedings.

23. M. Hirai et.al., Phys.Rev., D 69 , 054021 (2004).

24. M. Gluck, E. Reya, M. Stratmann and W. Vogelsang, Phys.Rev.D63,094005,2001, hep-ph/0011215

25. N. Bianchi, A. Fantoni, and S. Liuti, Phys. Rev., D69, 014505 (2004) 


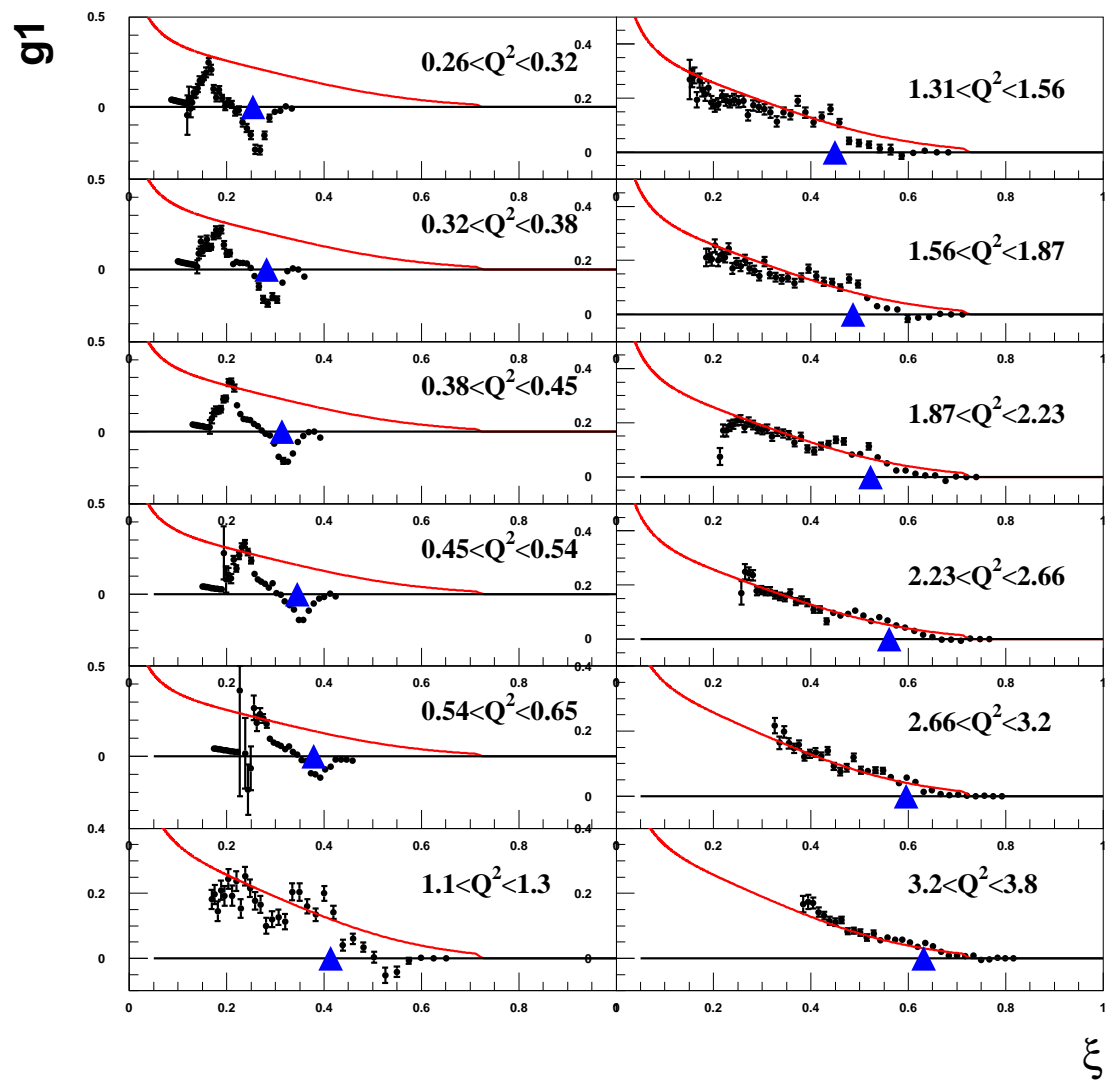

Figure 1. Measurements of the polarized structure function $\left(g_{1}\left(x, Q^{2}\right)\right)$ for the proton as extracted from the EG1 ${ }^{15} \mathrm{NH}_{3}$ data set. The line represents a fit to the world's DIS data at $Q^{2}=10 \mathrm{GeV}^{2} / \mathrm{c}^{2}$. 
aso

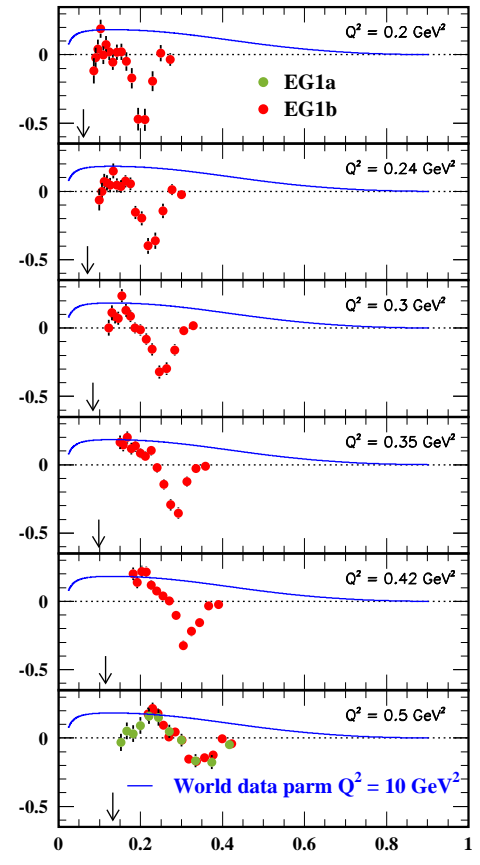

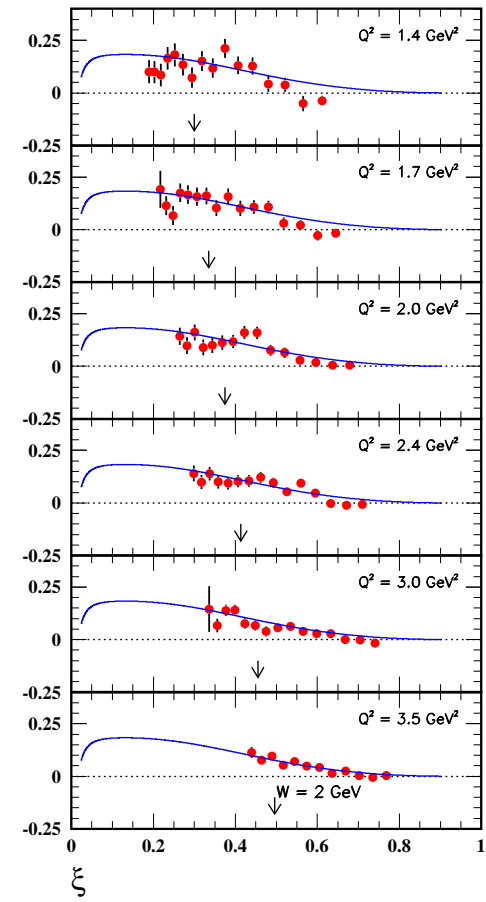

Figure 2. Measurements of the polarized structure function $\left(g_{1}\left(x, Q^{2}\right)\right)$ for the proton as extracted from the EG1 ${ }^{15} \mathrm{ND}_{3}$ data set. The arrow indicates the kinematic location of the conventional DIS domain $(W>2.0 \mathrm{GeV}$. The line represents a fit to the world's DIS data at $Q^{2}=10 \mathrm{GeV}^{2} / \mathrm{c}^{2}$. 


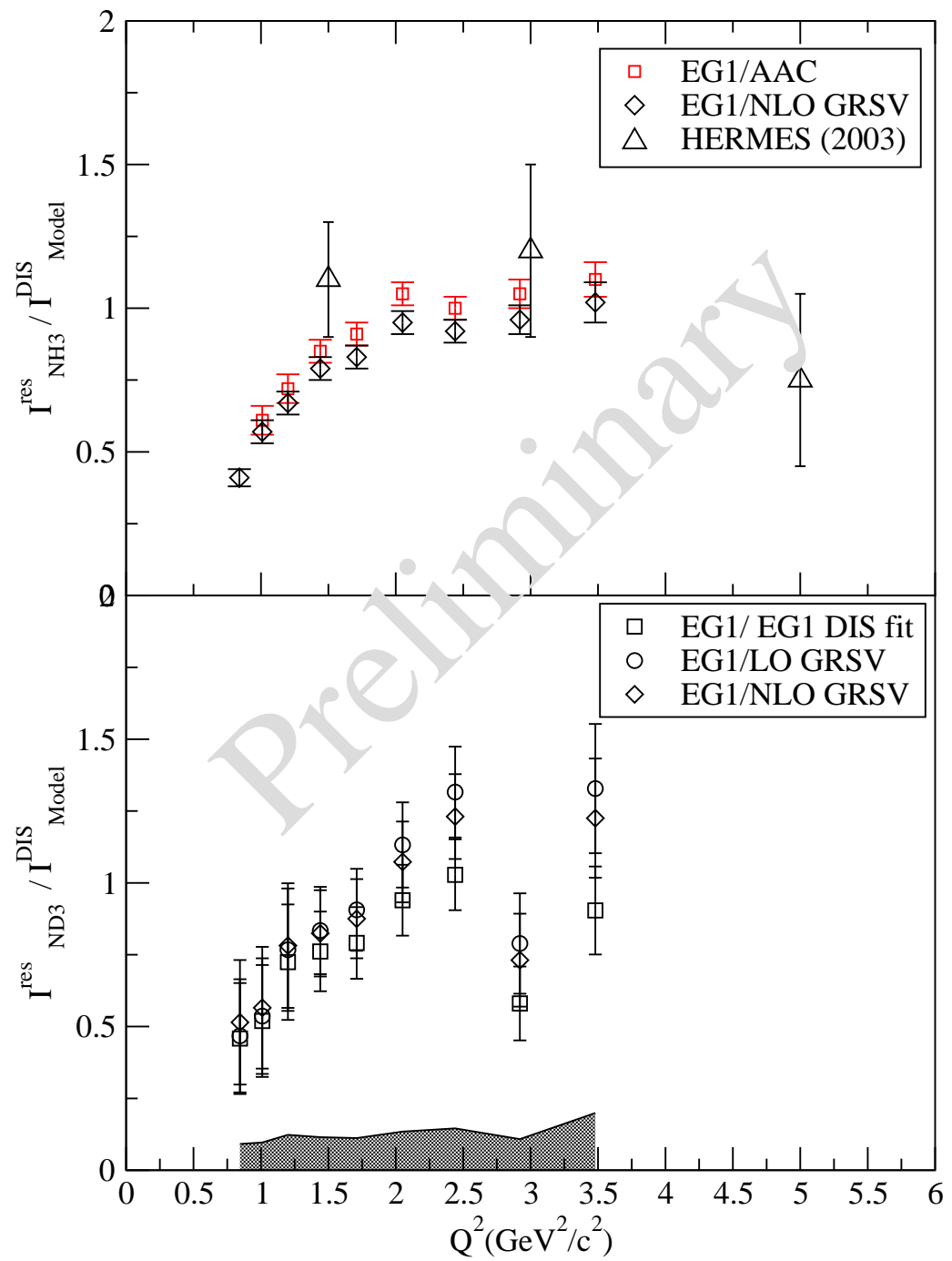

Figure 3. The ratio of the first moment of $g_{1}\left(x, Q^{2}\right)$ measured in the resonance region $\left(I^{r e s}\right)$ to the first moment of a global fit to $g_{1}\left(x, Q^{2}\right)$ in the deep inelastic region $\left(I_{M \text { odel }}^{D I S}\right)$ for several different $Q^{2}$ values. Both integrals are over the same kinematic region in $x$. Neither integral included elastic contributions. ( $\left.I_{\text {Model }}^{D I S}\right)$ was evaluated using fits to the world data set given by $\mathrm{EG}^{19}, \mathrm{AAC}^{23}$ and the GRSV 24 . 